\section{Global burden of depression: the intersection of culture and medicine ${ }^{\dagger}$}

\author{
JAN SCOTT and BARBARA DICKEY
}

The statistics about depression clearly identify it as a major public health problem (Greenberg et al, 1993). About 6\% of the population meet the criteria for major depressive disorder or dysthymia at any time, and $20 \%$ of those with major depressive disorder will have symptoms that persist beyond 2 years (Keller et al, 1992). The disorder is highly recurrent; $30 \%$ of individuals experience a relapse within 3 months of recovery and (in the absence of continuation or maintenance treatment) $50 \%$ experience a further episode within 2 years. The standardised mortality ratios for unipolar depression for accidental deaths, for deaths by natural causes and for suicide were 1.4, 1.7 and 19.7, respectively (Ustun, 1999). In the National Health Service the cost of treating depression ( $£ 887$ million) exceeds the cost of treating both hypertension ( $£ 439$ million) and diabetes $(£ 300$ million) (Department of Health, 1996). However, the direct health care costs are dwarfed by the indirect costs (Berndt et al, 2000). Days lost from work owing to depression exceed all other disorders and the economic burden on family members and society is considerable (Broadhead et al, $1990)$. This may account for $60-85 \%$ of the total cost of the illness and represents a significant proportion of the gross national product (Kind \& Sorensen, 1993; World Health Organization, 2001).

Although these data have been well known within psychiatry for many decades, they had only limited impact on the perceptions of depression within general medicine and by the public at large (Hays et al, 1995). This changed with the World Bank publication on the global burden of disease (Murray \& Lopez, 1996). In many ways the World Bank report was a watershed for psychiatry. It provided a meaningful way of communicating, to a wider audience, the true global impact of

†See pp. 12I-131, this issue. depression but it also emphasised to psychiatrists the salience of economic evaluations of the cost of treatment of mental disorders. The report employed a single standard construct, named the DALY (disability-adjusted life-year), to quantify objectively the disease burden. It demonstrated that in individuals aged 15-44 years depression accounted for $10 \%$ of all DALYs. Furthermore, this figure is projected to rise to $15 \%$ by the year 2020 , making depression second only to ischaemic heart disease in terms of worldwide disease burden. The report highlighted that investments in improving health are essential for economic development, and stated that we need to know both the cost and magnitude of gains that can be expected through improved primary, secondary or tertiary prevention of depression (Sturm \& Wells, 1995).

Research practice has reflected this increased interest in health economics, and many randomised controlled trials (RCTs) now include a cost-effectiveness analysis. However, there are problems in using these data alone to estimate the cost of depression. First, RCT research protocols limit or eliminate the choices that individuals make about treatment to reduce the variance in clinician and patient behaviours (Scott \& Sensky, 2003). Second, individuals with depression and concurrent physical or mental disorders are rarely included in RCTs (only $13 \%$ of depression studies include these subjects), whereas such comorbidity is reported in $50-70 \%$ of cases of depression seen in general practice (Katon \& Sullivan, 1990; Kessler et al, 1996). Third, primary care physicians treat more individuals with depressive symptoms than are formally diagnosed as having major depressive disorder (Johnson et al, 1992). Again, the former are less likely to be included in treatment studies. Consequently, economic analyses from RCTs of depression tell us about the cost-effectiveness of which treatment the individual received
(Lave et al, 1998). However, in clinical practice the underrecognition and undertreatment of depression in primary care (only $30-40 \%$ of individuals with major depressive disorder receive treatment) means that a more critical economic question is the cost that depends on whether the individual gets any treatment or not (Wells et al, 1996).

\section{THE LIDO STUDIES}

The Longitudinal Investigation of Depression Outcomes (LIDO) project is an important cross-national observational study exploring the relationship between symptom severity, quality of life, level of functioning, costs of treatment and outcome of depression. The paper by Chisholm et al (2003, this issue) builds on previous reports of treated cases of depression (e.g. Herman et al, 2002). Chisholm et al focus on a cohort of individuals attending primary care services in six different cities who, despite meeting the criteria for either major depressive disorder or sub-threshold depression, remained untreated for at least 3 months. The subjects were also assessed for the presence or absence of 12 chronic physical health problems, for comorbid anxiety or drug and alcohol misuse and on the SF-12 (a measure of subjective health status).

The paper by Chisholm et al makes fascinating reading. The first finding to note is the proportion of cases in the different subgroups: although the number of major depressive disorder $(n=1193)$ and sub-threshold cases $(n=1166)$ was very similar, the rates of comorbid depression (comorbid major depressive disorder, 21$43 \%$; comorbid sub-threshold depression, $21-35 \%)$ exceeded those of pure major depressive disorder $(11-25 \%)$ or pure subthreshold depression (12-28\%) at all sites. Second, the findings on cost were contrary to what we might reasonably predict. There were marked discrepancies in resource utilisation between sites but no differences in how providers approached the treatment of sub-threshold depression or major depressive disorder in any culture, and depression in the presence of a comorbid medical disorder led to higher resource use at only $50 \%$ of the sites.

The most intriguing finding was an association between the individual's perceived health status (on the SF-12) and the amount of health care they sought. At 
all six sites, it was self-reported health status rather than comorbidity that had a cost-raising influence on health care use. This supports previous research that subjective rather than objective levels of distress may dictate service use (Manning \& Wells, 1992; von Korff et al, 1992). Chisholm et al do not report on any relationship between severity of depression and self-reported health status, so we cannot determine whether individuals who are more depressed are less satisfied with their physical health, whether a subjective sense of poorer physical health increases the severity of depression or if the relationship is bidirectional. We can hypothesise that any association between depression and subjective and objective physical status may be causal, non-causal or independent, which may result in additive, multiplied or exponential increases in the cost of care (Sturm \& Wells, 1995; Wells et al, 1996).

Obviously the LIDO studies cannot provide answers to all our questions and we can only speculate about how the pattern and variation in resource utilisation reflects the characteristics of the different health care delivery systems. For example, it can be hypothesised that the small differences between subgroup treatment costs (as in St Petersberg) are indicative of lessindividualised approaches to treatment in that culture. In contrast, in Seattle there is an incremental increase in treatment costs in the three comorbid subgroups, which may suggest a highly differentiated approach to treatment.

\section{IMPLICATIONS}

There is a paucity of information on the economic consequences of depression in an international context (Ormel et al, 1994). Therefore, the LIDO studies on cross-cultural similarities and differences in health care utilisation and the lost human capital associated with depression deserve attention. The economic burden of untreated depression is graphically illustrated by the fact that at four of the six research sites the total health care consumption per subject approached, or was greater than, the average per capita health care expenditure in that country. Chisholm et al describe the cost of depression but cannot relate this to clinical outcome. Consequently, the LIDO study does not identify which, if any, of the services represent value

JAN SCOTT, MD, FRCPsych, Department of Psychological Medicine, Institute of Psychiatry, London; BARBARA DICKEY, PhD, Department of Psychiatry, Harvard Medical School, The Cambridge Hospital, Cambridge, Massachussets, USA

Correspondence: Jan Scott, Department of Psychological Medicine, PO Box 96, Institute of Psychiatry, De Crespigny Park, London SE5 8AF, UK

(First received 19 December 2002, final revision 31 March 2003, accepted 2 April 2003)

for money (Sturm \& Wells, 1995). Previous research in the USA suggests that successful treatment of an acute episode of depression is more expensive (about $30-50 \%$ higher cost) but more cost-effective than inadequate treatment, but these findings only apply to cases meeting the criteria for major depressive disorder (McCoombs et al, 1990; Simon et al, 1995). There is no robust evidence regarding the clinical effectiveness or cost-effectiveness of treatments for sub-threshold depression (Agency for Health Care Policy and Research, 1993; Lave et al, 1998). This may not be a major issue for health economists, because research demonstrates that more severe episodes of major depressive disorder are more likely to be treated. It is argued that strengthening the capacity of any primary care system to identify and treat these cases will theoretically have an impact on the overall burden of disease (von Korff $e t a l$, 1998). However, there are clinical drawbacks to applying this strategy in isolation. For example, $80 \%$ of individuals with subthreshold symptoms will go on to experience an episode of major depressive disorder (Judd et al, 1998), so we need information about any longer-term benefits of treating these cases. Most importantly, there is a need to produce a shift in treatment strategies for depression. The clinical and health-economic focus on the management of discrete episodes of major depressive disorder is outdated and there is a need to treat depression as a chronic disorder characterised by recurrent episodes over a lifetime (Scott et al, 2002). If depression is viewed in this way, the most costeffective treatment will be the one that prevents relapse. Given the increasing global burden of depression, we suggest that a crucial next step in research is to identify cross-national similarities and differences in the costs and long-term prognosis of depression.

\section{DECLARATION OF INTEREST}

None.

\section{REFERENCES}

Agency for Health Care Policy and Research (1993) Treatment of Major Depression: Clinical Practice Guidelines. no. 5, vol. 2. Rockville, MD: US Department of Health \& Human Services.

Berndt, E., Koran, L., Finkelstein, S., et al (2000) Lost human capital from early onset chronic depression. American Journal of Psychiatry, 157, 940-947.

Broadhead, W., Blazer, D., George, L., et al (1990) Depression, disability days and days lost from work in a prospective epidemiological study. Journal of the American Medical Association, 264, 2524-2528.

Chisholm, D., Diehr, P., Knapp, M., et al (2003) Depression status, medical comorbidity and resource costs. Evidence from an international study of major depression in primary care (LIDO). British Journal of Psychiatry, 183, 121-131.

Department of Health (1996) NHS Executive Report: Burdens of Disease. Leeds: Department of Health.

Greenberg, P., Stiglin, L., Finkelstein, S., et al (1993) The economic burden of depression in 1990. Journal of Clinical Psychiatry, 54, 405-418.

Hays, R., Wells, K., Sherbourne, C., et al (1995) Functioning and well-being of patients with depression compared with chronic general medical illness. Archives of General Psychiatry, 52, II-19.

Herman, H., Patrick, D., Diehr, P., et al (the LIDO Group) (2002) Longitudinal investigation of depression outcomes in primary care in six countries: the LIDO study - functional status, health service use and treatment of people with depressive symptoms. Psychological Medicine, 32, 899-902.

Johnson, J., Weissman, M. \& Klerman, G. (1992) Service utilization and social morbidity associated with depressive symptoms in the community. Journal of the American Medical Association, 267, 1478-1483.

Judd, L., Akiskal, H. \& Maser, J. (1998) Major depressive disorder: a prospective study of residual subthreshold depressive symptoms as a predictor of rapid relapse. Journal of Affective Disorders, 50, 97-108.

Katon, W. \& Sullivan, M. (1990) Depression and chronic medical illness. Journal of Clinical Psychiatry, 5I, $3-14$.

Keller, M., Lavori, P., Mueller, T., et al (1992) Time to recovery, chronicity and levels of psychopathology in major depression: a 5 year prospective follow-up of 431 subjects. Archives of General Psychiatry, 49, 809-816.

Kessler, R. C., Nelson, C. B. \& McGonagle, A. K. (1996) Comorbidity of DSM-III-R major depressive disorder in the general population: results from the US National Comorbidity Survey. British Journal of Psychiatry, 168 (suppl. 30), 17-30.

Kind, P. \& Sorensen, J. (1993) The costs of depression. International Clinical Psychopharmacology, 7, 191-195.

Lave, J., Schulberg, H. \& Kamlet, M. (1998) Costeffectiveness of treatment for depression in primary care practice. Archives of General Psychiatry, 55, 645-65I. 
Manning, W. \& Wells, K. (1992) The effects of psychosocial distress and psychological well-being on use of medical services. Medical Care, 30, 54I-553.

McCoombs, J., Nichol, M., Stimmel, G., et al (1990) The cost of antidepressant drug therapy failure: a study of antidepressant use patterns in a Medicaid population. Journal of Clinical Psychiatry, 51, 60-69.

Murray, C. \& Lopez, A. (1996) The Global Burden of Disease. Cambridge, MA: Harvard University Press.

Ormel, J., von Korff, M., Ustun, T., et al (1994)

Common mental disorders and disability across

cultures. Journal of the American Medical Association, 272, $174 \mid-1748$.
Scott, J. \& Sensky, T. (2003) Randomized controlled trials for psychological problems in primary care. Psychological Medicine, 33, 191-196.

—, Thorne, A. \& Horn, P. (2002) Effect of a chronic disease management approach to detection and treatment of depression in primary care. $B M$ J, $\mathbf{3 2 5}$ 951-954

Simon, G., von Korff, M. \& Barlow, W. (1995) Health care costs of primary care patients with recognized depression. Archives of General Psychiatry, 52, 850-856.

Sturm, R. \& Wells, K. (1995) How can care for depression become more cost-effective? Journal of the American Medical Association, 273, 5I-58.

Ustun, T. (1999) Global burden of mental disorders. American Journal of Public Health, 89, 1315-1318. von Korff, M., Ormel, J., Katon, W., et al (1992)

Disability and depression among high utilizers of health care. A longitudinal analysis. Archives of General Psychiatry, 49, 91-100

_, Katon, W., Bush, T., et al (1998) Treatment costs, cost offset and cost-effectiveness of collaborative management of depression. Psychosomatic Medicine, 60, 143-149.

Wells, K., Sturm, R., Sherbourne, C., et al (1996) Caring for Depression. A RAND Study. Cambridge, MA: Harvard University Press.

World Health Organization (200I) The World Health Report 2001: Mental Health: New Understanding, New Hope. Geneva: World Health Organization. 